Ann. Sc. forest., 1980, 37 (2), 147-157

\title{
Implantation artificielle du gui (Viscum album L.) sur différentes provenances de sapin pectiné (Abies alba Miller)
}

\author{
H. FROCHOT $\left({ }^{1}\right)$ et L. LANIER $\left({ }^{2}\right)$ \\ avec la Collaboration technique de Michel PITSCH ( $\left.{ }^{1}\right)$ \\ (1) Station de Sylviculture \\ Centre national de Recherches forestières, I.N.R.A., \\ Champenoux, 54280 Seichamps \\ ${ }^{2}{ }^{2}$ Chaire de Sylviculture, E.N.G.R.E.F.. \\ 14, rue Girardet \\ 54042 Nancy Cedex
}

\begin{abstract}
Résumé
Des essais d'implantation de gui du sapin (Viscum album L.) sont réalisés près de Nancy (France) sur des jeunes sapins pectinés de 10 ans issus de quatre régions géographiquement distinctes. Les réussites d'implantation (environ 12 p. 100 des baies déposées), montrent :

1) l'intérêt de la technique expérimentée sur des jeunes plants ;

2) l'absence de résistance au gui - dans les conditions de la Lorraine - du sapin issu de Normandie, pourtant totalement indemne dans sa région d'origine.
\end{abstract}

Le gui du sapin, très abondant dans les massifs montagneux français, présente une répartition irrégulière qui demeure encore inexpliquée.

Une liaison avec le vieillissement et l'altitude des peuplements de sapin a été démontrée par Peter-Contesse, Plagnat, Brossier. Mais, malgré de nombreuses observations, ces auteurs n'ont pas pu élablir de corrélation certaine avec d'autres facteurs écologiques.

Ne pourrait-on pas expliquer l'absence de gui du sapin de certaines régions ou stations par une incompatibilité de type génétique du sapin indigène avec le gui ?

Cette hypothèse, testée avec succès par Paine et Gäumann sur différentes variétés de poiriers, mérite d'être étudiée pour différentes provenances de sapin, comme le suggérait Lanier en 1969.

\section{1. - Généralités}

Sur le sapin en effet, de nombreuses observations (Brossier, 1969, Brossier, Plagnat, 1960, Lanternier, 1944, Peter-Contesse, 1930, Peter-Contesse, 1937, Plagnat, 1950, Plagnat, 1969) ont montré la liaison étroite entre le vieillissement des peuplements et le degré d'attaque par le gui. Sur les sujets âgés, le gui augmente le nombre 


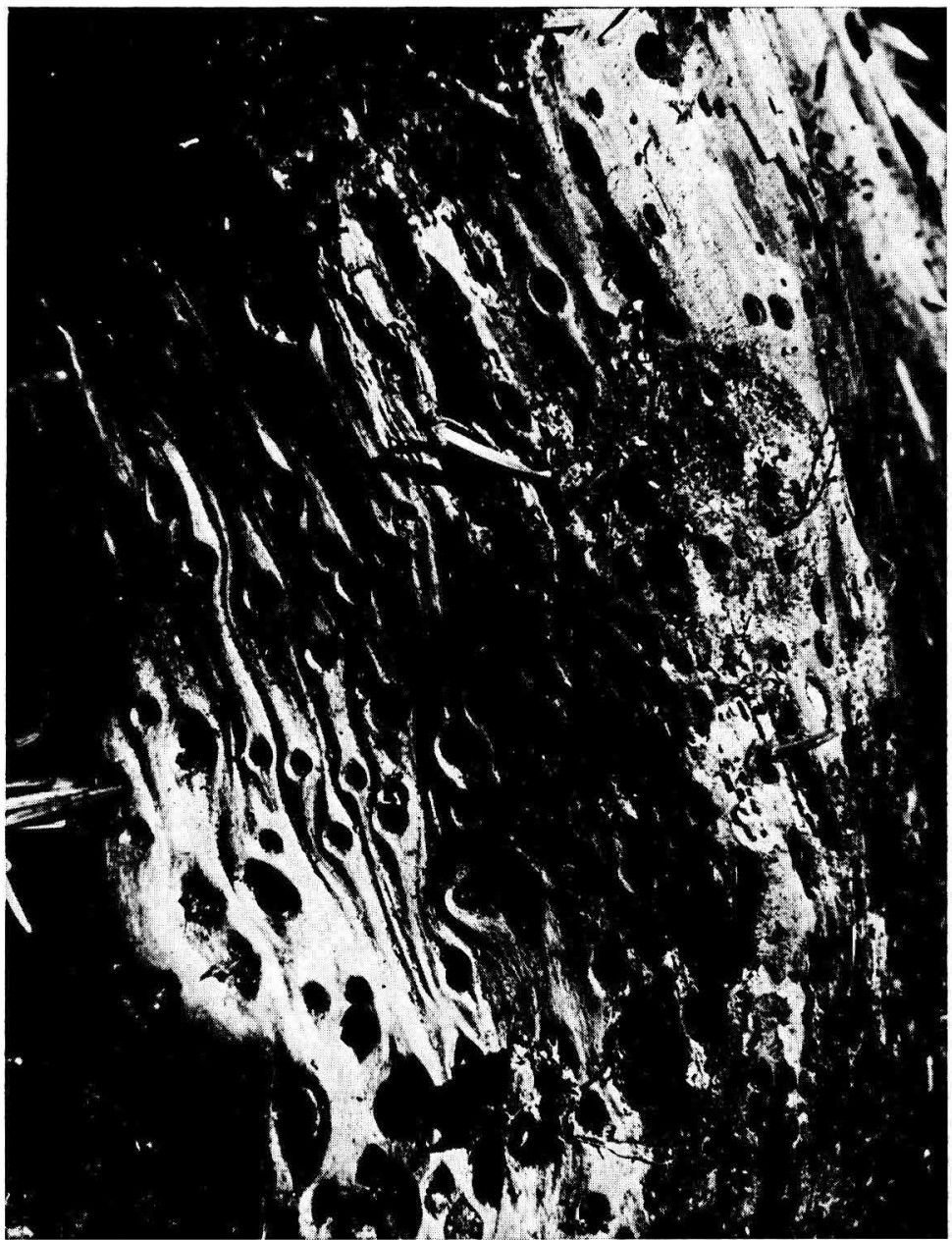

PHOTO No 1 (Cliché L. Lanier)

Dégât de gui sur sapin pectiné. On voit l'emplacement des suçoirs qui ont disparu. Mistletoe damages upon silver fir. The sinker traces are visible.

de ses suçoirs secondaires, entraînant des dégâts technologiques importants (photo $n^{\circ}$ 1). L'arbre est privé d'une partie de plus en plus grande de sa sève et ne s'accroît plus en diamètre : Peter-Contesse (Peter-Contesse, 1937) a mesuré, sur des arbres très atteints, un accroissement moyen en diamètre de $1 \mathrm{~cm}$ seulement en 20-30 ans. Enfin, l'installation du gui dans la cime entraîne souvent le dessèchement de la flèche, rapidement suivi de la mort de l'arbre. Ces observations conduisent, surtout lorsque les conditions deviennent proches des frontières écologiques, à limiter les révolutions à la centaine d'années, parfois moins.

De même, ces forestiers ont montré que l'importance des attaques du gui diminue au fur et à mesure que l'altitude augmente. En effet, lorsque le sapin descend par «avalaison » en dessous de sa limite altitudinale climacique (600 m par exemple en 


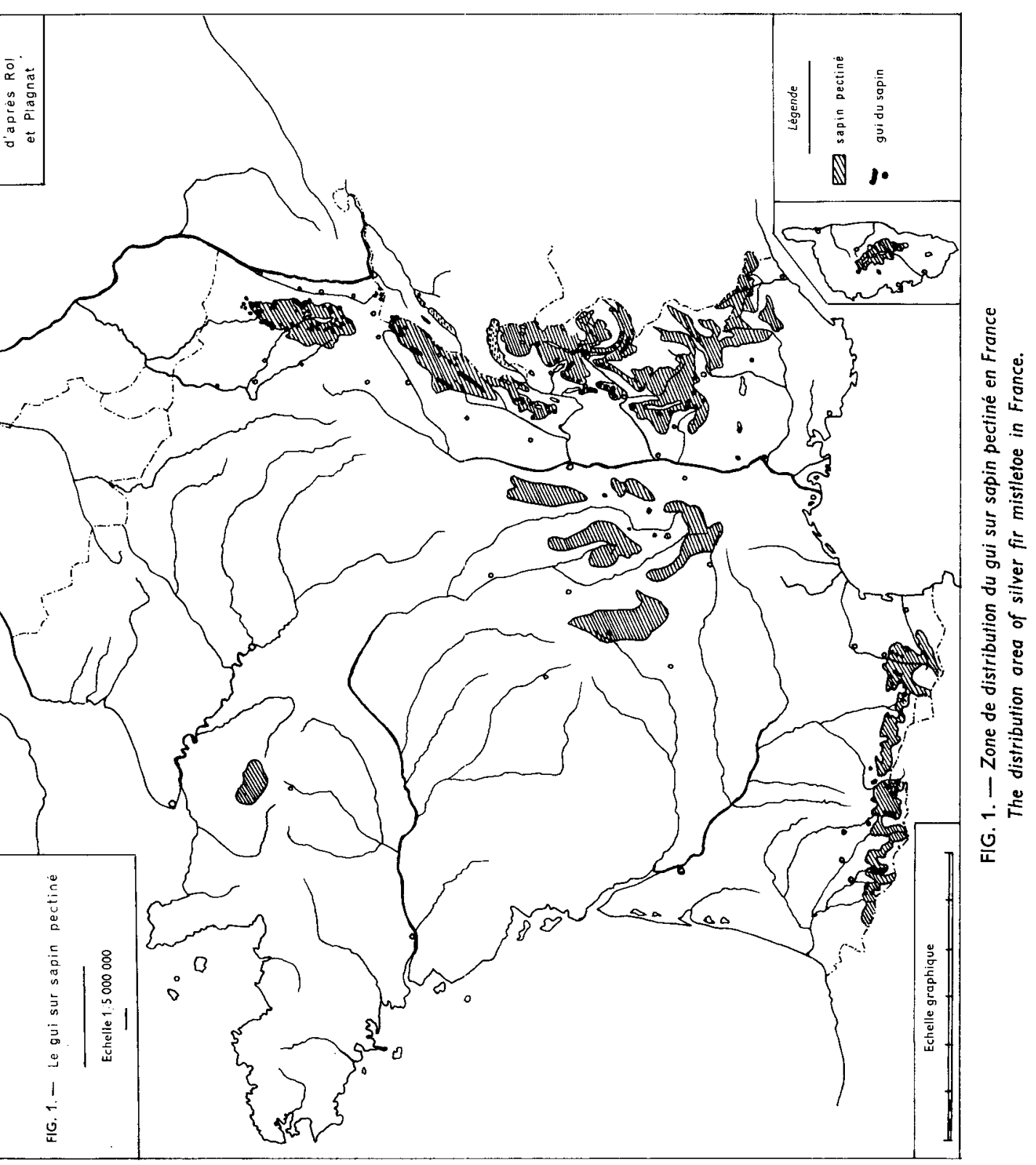


Chartreuse, selon Rol, (Rol, 1937)), il est très fortement atteint ; au contraire, en altitude, les attaques deviennent rares et semblent bequcoup mieux supportées. Ceci conduit à éviter d'introduire le sapin trop en dessous de son optimum écologique montagnard. Mais, il existe aussi de nombreuses sapinières guitées « bien en station » (Plagnat, 1950), pour lesquelles on ne connaît actuellement pas d'autres solutions, mise à part la lutte chimique (Delabraze, Lanier, 1972), que le rajeunissement des peuplements atteints ef la substitution d'essences.

Les sapinières normandes à 250-320 m (Rol, 1937) au-dessus du niveau de la mer, en situation marginale, devraient selon la théorie précédente être fortement attaquées. Or, il est bien connu, qu'elles sont totalement indemnes de gui.

On peut penser que cet îlot de sapin, éloigné de $350 \mathrm{~km}$ des sapinières guitées les plus proches (fig. 1) n'a jamais été contaminé par le gui*. En effet, les oiseaux qui véhiculent le parasite (principalement la grive draine, Turdus viscivorus L.), volant pendant leur migration à environ $45 \mathrm{~km} / \mathrm{h}$, ont évacué les graines de gui de sapin avant leur arrivée éventuelle en Normandie (ll faut en effet $1 / 2 \mathrm{~h}$ au plus pour qu'une grive évacue des graines ingérées (Tubeuf Von, 1923, Heim de Balzac, Mayaud, 1930)).

Mais, il peut y avoir des infestations accidentelles : baies collées aux pattes des oiseaux, transport par l'homme... On ne peut donc pas rejeter a priori l'hypothèse d'une incompatibilité entre le sapin de Normandie et le gui : soit que le sapin, du fait des conditions écologiques, réagit et entraîne la mort des plantules de gui par nécrose des tissus corticaux (selon la théorie de Gäumann, (Gäumann, Peter-Contesse, 1951)) ; soit qu'il existe une résistance de type génétique de la race normande de sapin au gui (selon l'hypothèse de Guinier (Guinier, 1934)).

II faut noter enfin, à ce propos, l'absence presque totale de données sur le comportement et les réactions du sapin pectiné lors de l'implantation des baies de gui : le parasite pénètre-t-il facilement dans l'écorce ? L'âge du rameau ou du sapin sur lequel il s'installe lui est-il indifférent? La faculté du sapin de se laisser parasiter par le gui est-elle un facteur constant au cours de la vie d'un arbre, ou bien y-a-t-il une sensibilisation liée au degré d'attaque par le gui (Paine, 1950) ? La lumière, considérée depuis longtemps comme un facteur déterminant pour le développement du gui, a-t-elle une influence sur le développement de la plantule?

Pour répondre à quelques unes de ces questions, nous avons entrepris des expériences d'implantation de gui sur de jeunes sapins pectinés de différentes provenances rassemblées dans les conditions de la Lorraine.

\section{2. - Matériel et méthode}

L'étude a été réalisée dans une place d'expérience préalablement installée par la station d'amélioration du C.N.R.F. ; elle est située en forêt d'Amance, à $15 \mathrm{~km}$ au N.E. de Nancy (France), sur pélosol assaini par des fossés de drainage. Les sapins (Abies alba Miller, $2+3$ ), issus de graines traitées par la station d'amélioration ef élevés à la pépinière du C.N.R.F., ont été repiqués au printemps 1968 en placeaux de quarante individus chacun.

* Il existe trois variétés de gui qui parasitent spécifiquement des hôtes bien précis (Tubeuf, Von, 1923). Le sapin n'est pas parasité par le gui du pin, ni par le gui des feuillus, mais uniquement par le gui du sapin. 
Les provenances de sapin

On a choisi pour l'expérimentation les provenances disponibles, bien portantes, qui représentaient aussi exactement que possible l'aire du sapin pectiné en France : deux provenances normandes, issues de la seule région française totalement indemne de gui, sont comparées à autant de provenances du Massif Central et des Pyrénées. Deux provenances des Balkans ont également été utilisées.

TABLEAU 1

Quelques caractéristiques des provenances de sapin pectiné (Albies alba)

Some characteristics of different provenances of silver fir (Abies alba)

\begin{tabular}{|c|c|c|c|c|c|c|c|c|}
\hline \multirow[b]{2}{*}{ Origine } & \multicolumn{8}{|c|}{ Dénomination } \\
\hline & $\begin{array}{c}\text { Ecouve } \\
\text { II }\end{array}$ & Perseigne & $\begin{array}{c}\text { Bois } \\
\text { noirs II }\end{array}$ & Mollières & Puivert & Rialsesse & Prahovei & Rhodope \\
\hline Pays & \multicolumn{6}{|c|}{ France } & $\begin{array}{c}\text { Rouma- } \\
\text { nie }\end{array}$ & Bulgarie \\
\hline Région & \multicolumn{2}{|c|}{ Normandie } & \multicolumn{2}{|c|}{ Massif Central } & \multicolumn{2}{|c|}{ Pyrénées } & \multicolumn{2}{|c|}{ Balkans } \\
\hline $\begin{array}{l}\text { Localisation : } \\
\text { — commune } \ldots \ldots \\
\text { — département ..... }\end{array}$ & $\begin{array}{l}\text { Saint } \\
\text { Nicolas } \\
\text { de Bois } \\
(61)\end{array}$ & $\begin{array}{c}\text { Perseigne } \\
(72)\end{array}$ & $\begin{array}{c}\text { Chausse- } \\
\text { terre } \\
(42)\end{array}$ & $\begin{array}{c}\text { St Just } \\
\text { d'Avray } \\
(69)\end{array}$ & $\begin{array}{l}\text { Puivert } \\
\text { (11) }\end{array}$ & $\begin{array}{l}\text { Arques } \\
\text { (11) }\end{array}$ & $\begin{array}{c}\text { Vallée } \\
\text { de } \\
\text { Prahovei }\end{array}$ & $\begin{array}{c}\text { Monts } \\
\text { Rhodope }\end{array}$ \\
\hline Altitude $(\mathrm{m}) \ldots \ldots \ldots$ & 380 & $2-300$ & 850 & 800 & $7-900$ & $6-700$ & 1100 & $?$ \\
\hline Pluviométrie $(\mathrm{mm}) \ldots$ & 846 & 770 & 1139 & 939 & 1041 & 969 & $800 / 900$ & ? \\
\hline
\end{tabular}

Technique d'inoculation du gui

L'infestation naturelle se fait à partir de baies mûres de gui (Tubeuf Von, 1923, Heim de Balzac, Mayaud, 1930), qui contiennent une graine possédant une ou plusieurs plantules prêtes à germer. Un certain nombre de baies, tombant d'une boule de gui en position haute, éclatent* sur des branches plus basses; la graine se colle, ef les plantules entament leur processus de développement (dissémination verticale). D'autres sont collées sur des rameaux par la fauvette à tête noire (Sylvia atricapilla L.) qui dégarnit la graine de sa partie comestible, la viscine ; d'autres enfin sont avalées par les grives draines (Turdus viscivorus L.) qui en sont très friandes ; les graines, éjectées avec les fientes, sont disséminées dans l'espace au hasard des allées ef venues de ces agents de transport (dissémination horizontale). II semble que ce dernier procédé soit le plus fréquent dans la nature. D'autres oiseaux participent probablement plus occasionnellement à cette dissémination.

L'implantation artificielle de gui est donc en théorie très simple : il suffit d'écraser sur l'écorce de l'arbre des baies bien mûres. La viscine (substance dont on fait la glu) contenue dans la baie permet à la graine d'adhérer au support, en attendant que le 
disque adhésif se fixe. Cette technique nécessite un assez grand nombre de baies, car il y a beaucoup de pertes ; elle a l'avantage d'être facile d'emploi.

\section{Dispositif expérimental}

Pour chaque provenance, on retient huit arbres.

Etant donné la rareté relative des baies de gui du sapin, et la faible quantité récoltée, une ou deux baies seulement sont affectées à chacun des arbres de l'essai.

Elles sont déposées sur l'écorce des branches des $3^{\mathrm{e}}, 4^{\mathrm{e}}$ et $5^{\mathrm{e}}$ verticilles, assez faciles d'accès et suffisamment développées. Pour réduire les risques de glissements, on choisit des emplacements favorables à la tenue des baies :

- soit au niveau de l'insertion de la branche sur le tronc,

- soit au niveau de l'insertion des ramifications latérales avec la branche, qui forme souvent un renflement à cet endroit.

Dans la mesure du possible, on recherche un bon éclairement, sans pour autant favoriser une exposition particulière.

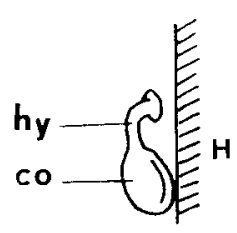

$+$

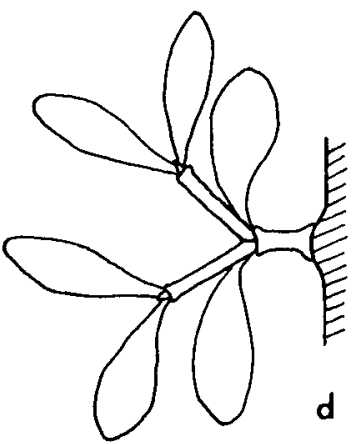

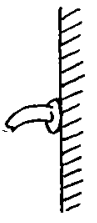

b

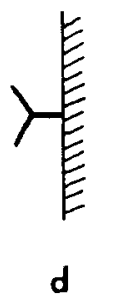

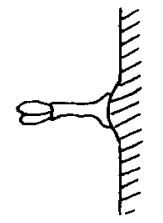

C

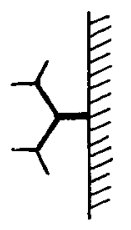

e

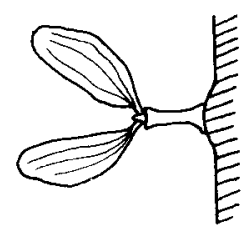

C

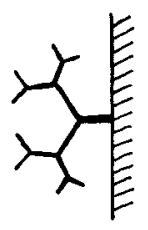

1

FIG. 2. - Stades de développement du gui.

The various development stages of mistletoe.

La graine adhère par sa partie cotylédonaire (co) d̀ l'écorce de l'hôte $(H)$. L'extrémité renflée de l'hypocotyle (hy) se rapproche de l'écorce par phototropisme négatif.

$a$. l'extrêmité renflée de l'hypocotyle établit le contact avec l'écorce de l'hôte et forme un cône de fixation (cf).

b. la partie cotylédonaire a disparu ; parfois l'hypocotyle disparaît également et il ne reste que le cône de fixation.

c. deux états du stade $c$ : premières feuilles naissantes, puis bien développées.

d. présence du deuxième étage d'articles : représentation à l'échelle, puis schématique.

e,f. présence de 3, 4 étages d'articles : représentation schématique. 
L'installation des baies a lieu deux années successives :

- les 22 et 23 mai 1973, cent vingt huit baies (seize par provenance) récoltées 6 jours avant en Chartreuse (forêt de Chapareillan, près de Grenoble, dans l'Isère) et conservées à la tempérałure extérieure.

- le 10 avril 1974, cent quatre baies (treize par provenance) récoltées dans les basses Vosges (forêt de Badonviller, en Meurthe-et-Moselle) et conservées à $-10^{\circ} \mathrm{C}$ pendant 14 jours.

La température extérieure au moment de l'implantation était dans les deux cas $21,5 \circ \mathrm{C}$ maximum.

\section{Système de notation}

Les baies implantées sont repérées sur un plan et sur les sapins de façon à ne pas les perdre de vue, car la manifestation de la présence du gui, surtout dans son jeune âge, est très discrète.

Le système de notation s'appuie sur un schéma des stades de développement du gui sur son hôte (voir fig. 2), schéma que nous avons décrit et utilisé pour une étude sur la sensibilité du peuplier au gui (Frochot, Pitsch, Wehrlen, 1978).

\section{3. - Résultats}

Les implantations réalisées en 1973 n'ont donné aucun résultat positif, sans doute à cause de l'état déficient des baies récoltées en fin de saison sur arbres abattus (baies surmûres, avortées ou mal venantes).

Par contre, les cent quatre implantations réalisées en 1974 ont donné des résultats intéressants, qui sont présentés dans le tableau 2 (voir également photos 2 à 5).

\section{TABLEAU 2}

Résultats des implantations de gui (Viscum album L) sur différentes provenances de Sapin pectiné (Abies Alba) trois années après l'implantation des baies

Results of mistletoe (Viscum album) inoculations on different silver fir (Abies alba) provenances three years afters berries inoculation

\begin{tabular}{|c|c|c|c|c|c|c|c|c|}
\hline Dénomination & $\begin{array}{c}\text { Ecouve } \\
\text { II }\end{array}$ & Perseigne & $\begin{array}{l}\text { Bois } \\
\text { noirs II }\end{array}$ & Mollières & Puivert & Rialsesse & Prahovei & Rhodope \\
\hline Région & \multicolumn{2}{|c|}{ Normandie } & \multicolumn{2}{|c|}{ Massif Central } & \multicolumn{2}{|c|}{ Pyrénées } & \multicolumn{2}{|c|}{ Balkans } \\
\hline $\begin{array}{l}\text { Nombre d'implanta- } \\
\text { tions réussies ...... }\end{array}$ & 2 & 0 & 4 & 0 & 1 & 3 & 1 & 1 \\
\hline $\begin{array}{l}\text { Stades de développe- } \\
\text { menł (voir fig. } 2 \text { ) .... }\end{array}$ & $\begin{array}{l}d \\
e\end{array}$ & & $\begin{array}{l}2 d \\
2 \mathrm{e}\end{array}$ & & c & $\begin{array}{l}c \\
2 d\end{array}$ & $f$ & $\begin{array}{c}c \\
\text { (b) }\end{array}$ \\
\hline $\begin{array}{l}\text { Localisation des im- } \\
\text { plantations (1) ..... }\end{array}$ & $2 \mathrm{~B}$ & & $4 \mathrm{~B}$ & & $\mathrm{~T}$ & $2 \mathrm{~B}$ & B & $\begin{array}{l}B \\
(B)\end{array}$ \\
\hline Exposition $\ldots \ldots \ldots$ & $2 \mathrm{E}$ & & $\begin{array}{c}E, S E \\
W, N E\end{array}$ & & $E$ & $\underset{S}{2 \mathrm{SE}}$ & $\mathbf{E}$ & $\begin{array}{l}\text { SW } \\
(\mathrm{SE})\end{array}$ \\
\hline
\end{tabular}

(1) $\mathrm{B}=$ implantation sur une branche latérale.

$\mathrm{T}=$ implantation sur le tronc principal. 

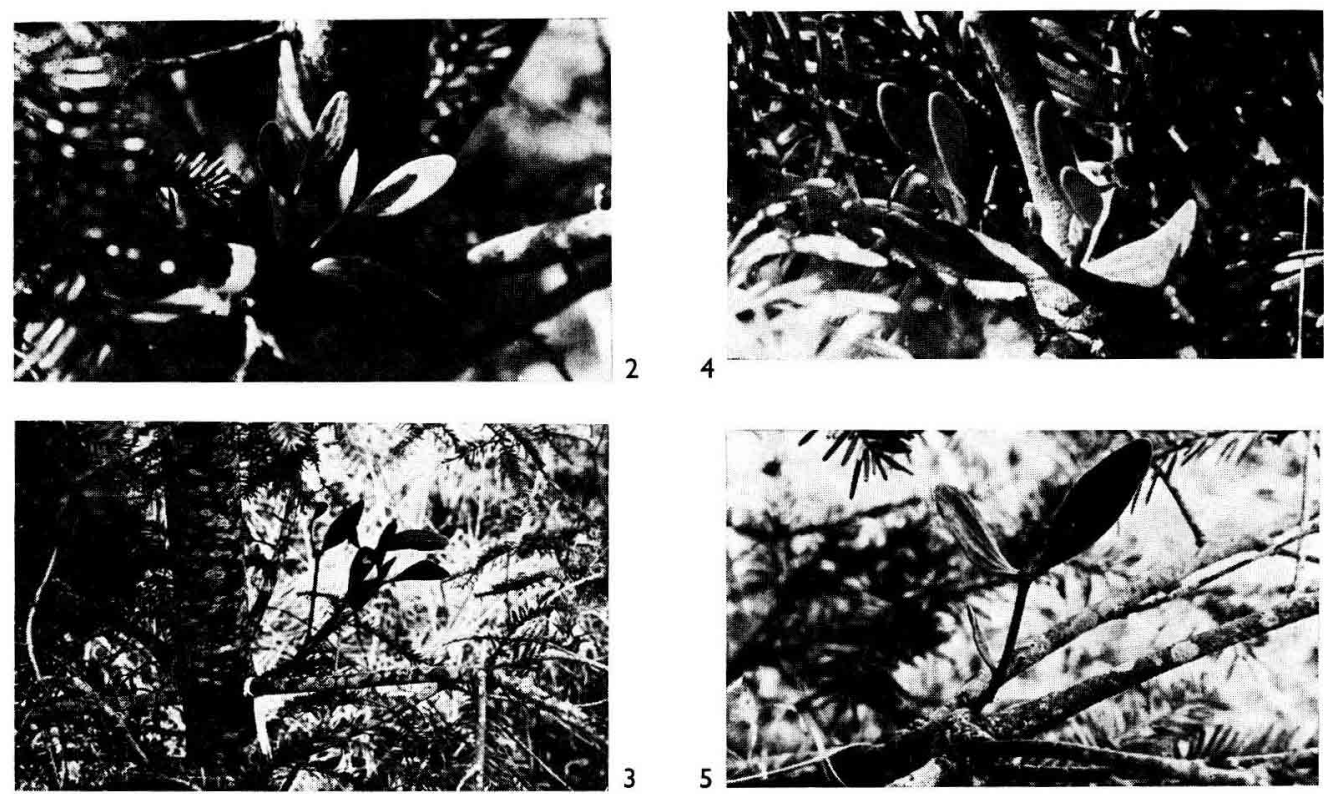

PHOTOS 2 à 5 (2-3 : Cliché L. Lanier ; 4-5 : Cliché L. Wehrlen.Développement de gui sur sapin pectiné

- En haut (2 et 4), provenance Ecouve (Normandie).

- En bas (3 et 5), provenance Bois noir (Massif Central).

L'installation de la baie date de :

- 3 années dans les photos 2 et 3 (stade d)

- 4 années dans les photos 4 et 5 (stade e, non ramifié en 5 ).

The berry inoculation has been made :

3 years ago (photos 2 and 3 : development stage d)

4 years ago (photos 4 and 5 : development stage e, without ramification for 5 ).

\section{4. - Discussion des résultats}

1) Le taux moyen de réussite, de 12 p. 100, peut paraître assez faible. Pourtant, si les graines disséminées naturellement s'implantaient avec autant de chances de réussite (environ une baie sur dix), ce serait un sucès remarquable pour l'expansion du gui. D'autre part, ce résultat est tout à fait comparable à ceux déjà obtenus sur les peupliers sensibles dans des conditions similaires : on trouve en effet 10,3 p. 100 pour Populus trichocarpa Fritzi Pauley ef 13,6 p. 100 pour Populus $\times$ euramericana 1214 (Frochot, Pitsch, Wehrlen, 1978).

2) Nous estimons avec Sallé (Sallé, 1975, Sallé, 1977, Frochot, Pitsch, Wehrlen, 1978) qu'à partir du stade c, l'implantation de gui est réussie : en effet, le stade a, caractérisé par la fixation du disque adhésif, ne nécessite pas un contact entre le gui et les tissus nourriciers de l'arbre. Il peut se réaliser sur n'importe quel support inerte. Le stade b - hypocotyle fixé, cotylédons absents - est l'étape pendant laquelle les tissus de gui pénètrent dans ceux de l'hôte. Sur peuplier comme sur sapin, nous avons observé que cette étape peut durer plusieurs années, et qu'elle peut évoluer 
aussi bien vers le dessèchement de l'hypocotyle que vers la naissance des deux premières feuilles du gui.

3) Le faible nombre de baies déposées (treize par provenance) nous interdit d'établir toute comparaison de sensibilité entre les différentes provenances. De même, l'échec constaté sur certaines provenances n'est pas une preuve absolue d'insensibilité.

4) L'expérimentation montre néanmoins qu'une provenance au moins de chacune des quatre régions comparées est susceptible d'être parasitée par le gui, dans les conditions de milieu de la Lorraine. On ne peut donc pas expliquer l'absence de gui des sapins de Normandie par une résistance purement génétique au gui.

Cette réponse fondamentale implique :

- qu'il ne faut surtout pas laisser s'installer le gui du sapin en Normandie,

- qu'il y a peu d'espoir de voir les introductions de sapins normands dans les zones sensibles des Vosges, du Jura, des Alpes, des Pyrénées, indemnes de gui, du fait de leur origine.

5) Tous les auteurs s'accordent à dire que le gui du sapin est un parasite de vieillesse. Les rares sapins de petite taille sur lesquels nous avons observé le gui dans les sapinières étaient toujours des arbres âgés situés en sous-étage. Or, nos réussites d'imslantation montrent que des sapins jeunes (10 ans) sont susceptibles d'être contaminés au même titre que des adultes. Le parasite peut également s'installer sur des rameaux ayant une année de végétation au moment du dépôt des baies.

Le fait que l'on trouve peu de gui dans les régénérations de sapins doit donc être attribué à d'autres causes qu'une résistance particulière due à la jeunesse : par exemple, le manque de lumière, ou tout simplement les faibles chances qu'ont les jeunes sapins d'avoir reçu naturellement les graines de gui, du fait de leur jeune âge, ef du faible volume qu'ils occupent, ou du fait du mode de vie des agents de transport ef des prédateurs.

6) Un certain nombre d'indications supplémentaires sur la biologie du parasite peuvent être données :

- la position dans l'espace de l'axe porteur semble sans influence sur la réussite de l'implantation :

- l'exposition Est à Sud-Est semble favoriser la réussite des implantations ; ceci est probablement en rapport avec le besoin en lumière du gui.

\section{Conclusion}

Les principaux enseignements de l'expérimentation que nous rapportons ici sont les suivants :

1) Absence de résistance naturelle au gui du sapin pectiné normand dans les conditions lorraines.

2) Réussite des implantations sur jeunes plants justifiant l'utilisation de cetfe technique, qu'il faudra améliorer en fonction des premiers résultats obtenus.

L'hypothèse d'incompatibilité de type génétique étant rejetée, restent les autres, auxquelles cette expérience n'a pas répondu. Une des plus probables semble être 
l'absence de graine de gui de sapin dans la région normande. Mais, il serait très imprudent de vouloir répondre à cette question par des implantations pratiquées en Normandie, même à titre expérimental.

Par contre, des expérimentations pourraient être réalisées avec précaution dans d'autres cas encore inexpliqués comme la présence d'îlots totalement indemnes en plein cœur d'une sapinière pyrénéenne ravagée par le gui. L'absence totale de graines semblant improbable dans ce cas, il faudrait tester d'autres hypothèses, notamment : les graines ne sont pas viables ou sont détruites par des agents naturels : le sapin réagit différemment aux implantations selon son état physiologique, ou en fonction de certains facteurs extérieurs.

Reçu pour publication en août 1979.

\author{
Summary \\ Artificial inoculation with mistletoe (Viscum album L.) \\ on different proveniences of silver Fir (Albies alba Miller)
}

Experiments of inoculation with mistletoe (Viscum album L.) were conducted near Nancy (France) on some young ( 10 years old) silver firs, coming from four different areas. The success of these inoculations (about 12 p. 100 of the deposited berries) showed:

1) the technical interest of the experiment on young plants,

2) the lack of resistance to mistletoe - in the Lorraine conditions - of the fir coming from Normandy, although totally undamaged in its origin area.

\title{
Références bibliographiques
}

BROSSIER J., 1969. Réflexions sur le gui du sapin, Rev. for. fr., XXI, 6, 558-561.

BROSSIER J., PLAGNAT F., 1960. Nouvelle sylviculture des sapinières à gui, Rev. for. fr., XII, 2 , 102-109.

DELABRAZE P., LANIER L., 1972. Contribution à la lutte chimique contre le gui (Viscum album L.), Eur. J. For. Pathol., 2, 2, 95-103.

FROCHOT H., PITSCH M., WEHRLEN L., 1978. Différences de sensibilité au gui (Viscum album L.) de quelques clônes de peuplier (Populus sp), « in »: 103e Congrès National des Sociétés Savantes, Nancy, I, 371-380.

GAUMANN E., PETER CONTESSE J., 1951. Neuere Erfahrungen über die Mistel, Schweizer. Z. Forstwes., 102, 2/3, 108-119.

GUINIER Ph., 1934. Les dommages causés par le gui, Ann. Sc. Natl. « Eaux ef Forêts », 19, $221-231$.

HEIM DE BALSAC H., MAYAUD N., 1930. Compléments à l'étude de la propagation du gui (Viscum album L.) par les oiseaux, Alauda, 1930, 7/8, 1-20.

LANIER L., 1969. Revue bibliographique et idées de recherche concernant les Loranthaceae, le Viscum album L. en particulier (mémoire de $2^{e}$ thèse Doc. Sc., C.N.R.F. Nancy, dactylogr., 80 p.).

LANTERNIER M., 1944. Le gui du sapin pectiné dans le département de la Savoie, Rev. « Eaux ef Forêts 》, 42, 460-469.

PAINE L., 1950. The succeptibility of pear trees to penetration and toxic damage by mistletoe, Phytopath. Zschr., 17, 305-327.

PETER-CONTESSE J., 1930. Du gui, J. For. Suisse, 81, 10, 217-223 et 11, 247-259.

PETER-CONTESSE J., 1931. Gui et possibilités, J. For. Suisse, 82, 10, 221-227.

PETER-CONTESSE J., 1937. Influence du gui sur la production de bois de service, J. For. Suisse, 88, 7, 145-153.

PLAGNAT F., 1950. Le gui du sapin, Ann. Ec. Natl. « Eaux et Forêts », XII, 1, 155-231.

PLAGNAT F., 1969. Sylviculture des sapinières à gui, conclusion provisoirement... définitive, Rev. For. Fr., XXI, 6, 553-557. 
ROL R., 1937. Contribution à l'étude de la répartition du sapin, Ann. Ec. Natl. " Eaux ef Forêts », VI, 2, 235-290.

SALLÉ G., 1975. Etude cytologique, cytochimique et histoautoradiographique du Viscum album L. (Loranthacées). I. La graine, sa germination et les modalités de la fixation sur l'hôte, Rev. Cyt. Biol. Végét., 1975, 38, 1-110.

SALLÉ G., 1977. Etude cytologique, cyłochimique et histoautoradiographique du Viscum album L. (Loranthacées), (Thèse de Doctorat d'Etat, Sc. Nat., Université Pierre et Marie Curie. Paris VI, 74 p. 88 fig.).

TUBEUF K. Von, 1923. Monographie der Mistel, München und Berlin, R. Oldenburg. 